Document downloaded from:

http://hdl.handle.net/10251/142955

This paper must be cited as:

Lechago-Buendia, S.; García Meca, C.; Griol Barres, A.; Kovylina, M.; Bellieres, LC.; Martí Sendra, J. (01-0). All-Silicon On-Chip Optical Nanoantennas as Efficient Interfaces for Plasmonic Devices. ACS Photonics. 6(5):1094-1099. https://doi.org/10.1021/acsphotonics.8b01596

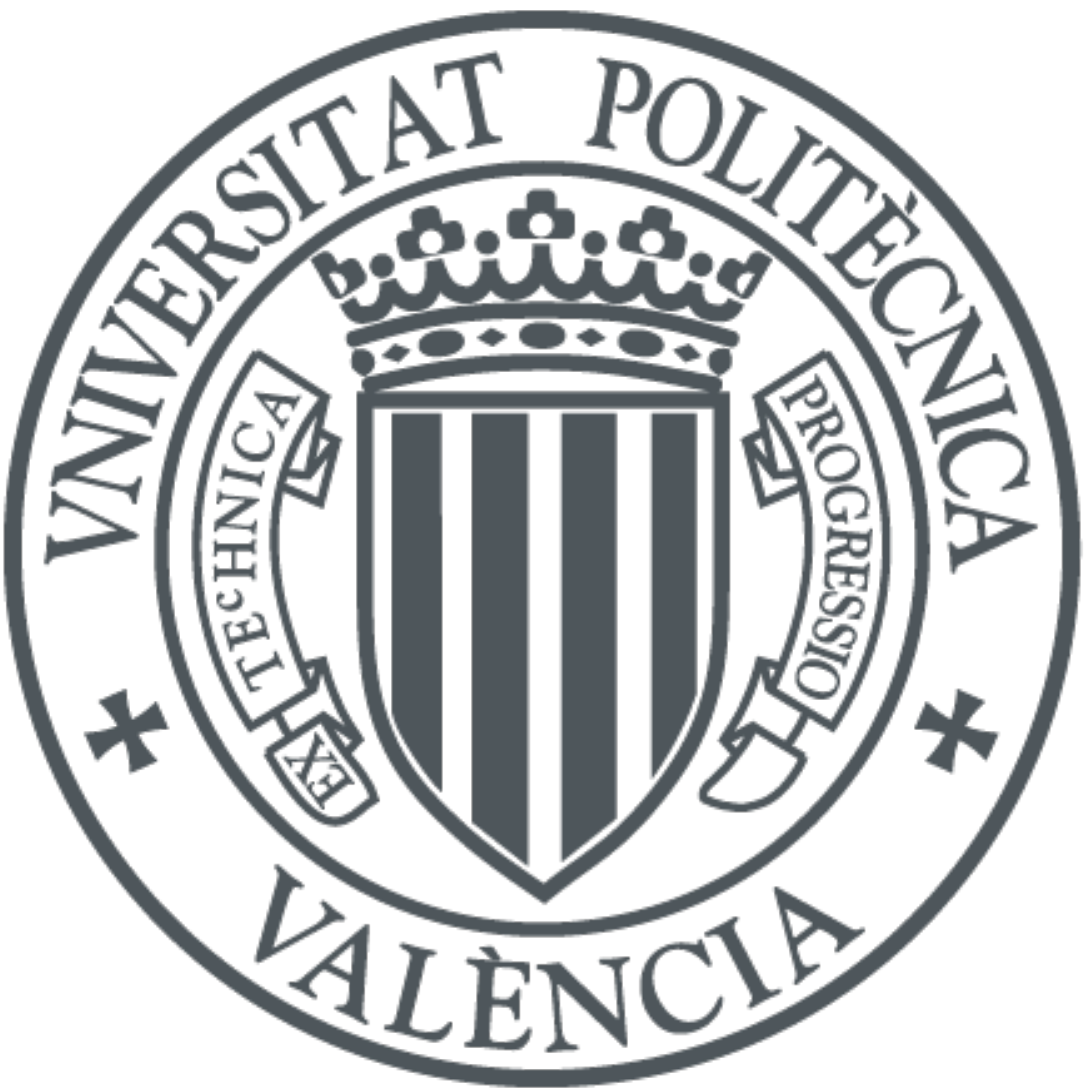

The final publication is available at

https://doi.org/10.1021/acsphotonics.8b01596

Copyright American Chemical Society

Additional Information 


\title{
All-Silicon On-chip Optical Nanoantennas As Efficient Interfaces for Plasmonic Devices
}

\author{
Sergio Lechago *, Carlos García-Meca, Amadeu Griol, Miroslavna \\ Kovylina, Laurent Bellieres and Javier Martí
}

\author{
Nanophotonics Technology Center, Universidad Politécnica de Valencia, Camino de Vera s/n, 46022 \\ Valencia, Spain. \\ *email: serlecbu@ntc.upv.es
}

KEYWORDS: Photonics, Integrated optics, Optical circuitry, Nanodevices, Nanoscale Interconnects.

\begin{abstract}
Plasmonic technology promises to unfold new advanced on-chip functionalities with direct applications in photovoltaics, light matter interaction and the miniaturization of optical interconnects at the nanoscale. In this scenario, it is crucial to efficiently drive light to/from plasmonic devices. However, typically-used plasmonic wires introduce prohibitive losses, hampering their use for many applications. Recently, plasmonic nanoantennas have been proposed to overcome this drawback, not only providing a notable loss reduction, but also an enhanced on-chip flexibility and reconfigurability. Nevertheless, these devices still perform poorly for long-reach interconnects, owing to their lowdirective radiation and low efficiency. Here, we introduce a class of slot-waveguide-based silicon nanoantennas that lift all these limitations, and show their feasibility to be connected directly and efficiently to plasmonic devices. To test the performance of these antennae, an on-chip plasmonic-dielectric interconnect is experimentally demonstrated over distances as high as $100 \mu \mathrm{m}$. In an outstanding manner, our wireless scheme clearly outperforms previous plasmonic approaches in terms of link efficiency and effective gain. This work paves the way to the development of ultra-fast onchip wireless reconfigurable and flexible interconnects, and additionally opens new avenues in optical manipulation and sensing applications.
\end{abstract}

Introduction: Plasmonic devices have enabled the development of important applications in fields such as spectroscopy, near-field optical microscopy or biosensing (1) thanks to their unique ability to engineer light at the nanoscale. Within integrated on-chip communications, plasmonic approaches offer the potential for the realization of ultra-compact low-cost devices (modulators, detectors or nanoscale chip sources (1)) able to perform at very high operation speeds with a low power consumption (2). The natural way for interconnecting these devices on the optical chip is the use of metallic nanowires. Nevertheless, guiding light over long distances via plasmonic waveguides results in prohibitive propagation losses (3), (4). An alternative to mitigate this loss relies on the use of dielectric couplers (5), able to interface plasmonic devices with typical silicon waveguides. In contrast, these dielectric-metallic wired schemes still provide stringent limitations in terms of flexibility and reconfigurability at the photonic layer (6). Recently, the emergence of plasmonic nanoantennas (7), (8), (9), (10) has also demonstrated their suitability to reduce propagation losses as compared to metallic nanowires (3). This allows, for instance, the implementation of unguided interconnects with interferenceless crossing paths on the same layer (thus avoiding multilayer geometries (9)), compact reconfigurable devices and more flexible on-chip networks (6), (10), (11). These features lessen the presence of waveguides, leading to simpler on-chip layouts (6), (11). Additionally, the interaction with the medium at far-field distances enabled by antennas, paves the way to less complex networks for communications (11) and new functionalities for sensing applications (6), (12), (13) and microparticle control (6), hardly achievable with wired-based 
approaches. However, while improving the performance of their wired counterparts, plasmonic nanoantennas yield low directivity values and high losses (10), (14) because of their strong field confinement and the metallic absorption in the optical regime (15), hindering their application for practical on-chip wireless links. On the other hand, nanoantennas based on dielectric nanoparticles (16), (17) have emerged, presenting better radiation efficiencies due to their low-loss nature and higher directivity than metallic structures, not always compromising the usual higher compactness of plasmonic approaches. Nevertheless, the complexity in the fabrication of these nanoparticles has prevented their practical implementation for wireless interconnects. Other approaches with enhanced directivities relies on the use of non-compact systems as dielectric arrays (18), (19), more suitable for off-plane interconnects.

More recently, it was demonstrated that most of the aforesaid disadvantages can be overcome by using stripwaveguide-based silicon nanoantennas, which have enabled the realization of long-reach dynamically-reconfigurable on-chip wireless interconnects, as well as of different lab-on-a-chip sensors (6), (13). It is then natural to ask whether these kind of dielectric antenna might interface plasmonic systems to boost a new variety of high-performance applications. However, although strip dielectric waveguides can be theoretically connected to slot plasmonic waveguides with a very high efficiency, this requires quite complex transitions between the plasmonic and dielectric structures, leading to a difficult error-sensitive implementation and large footprints, with a notable reduction of the experimental efficiency with respect to theoretical estimations (20).

In this letter, we explore a different wireless system built upon slot-waveguide-based silicon optical antennas able to be efficiently and directly connected to plasmonic slot wires. The simplicity of this dielectric-to-plasmonic transition allows us to obtain experimental coupling efficiencies surpassing those obtained with sophisticated strip-to-slot couplers. Through this hybrid design, we are able to combine the advantages provided by both dielectric and plasmonic technology to produce high-performance on-chip devices, boosting the development of new applications at the nanoscale.

Results and Discussion: As a starting point, we consider the system shown in Figure 1a. On the left, a semi-infinite plasmonic slot waveguide (PSW) is connected to a finite dielectric slot waveguide (DSW). With the suitable dimensions, the open end of the DSW behaves as an efficient aperture antenna(6), while the PSW acts as a bridge to connect this antenna with a plasmonic device. Therefore, a plasmonic mode guided by the PSW is converted to a guided mode in the subsequent DSW and radiated at its open end. An equal DSW-PSW hybrid device receives the radiated wave on the right and transforms it back to a plasmonic mode. It is worth mentioning that the plasmonic interconnect is not necessarily a long section of plasmonic wire, since a short waveguide section (with very low losses) would readily connect the wireless antennas with the plasmonic system for communications, sensing or other purposes. Our goal is to maximize the total power efficiency $\eta$ of the system, which is basically given by the product $\eta=\left(\eta_{\mathrm{C}} G R\right)^{2}$ for the proposed configuration. Here, $\eta_{\mathrm{C}}$ is the PSW-to-DSW coupling efficiency, $G$ is the gain of the DSW aperture antenna (including directivity, radiation efficiency and reflection coefficient) and $R=\lambda /(4 \pi d)$ accounts for the freespace propagation loss associated with a link distance $d$. The product $G_{\text {eff }}=\eta_{\mathrm{C}} G$ can be considered as the effective gain of the DSW-based antenna. Since $R$ is fixed, we need to find the optimal system geometrical parameters providing the combination of $\eta_{\mathrm{C}}$ and $G$ that maximizes $\eta$. Driven by technological feasibility, we constrained the height of all structures to $220 \mathrm{~nm}$, assuring their compatibility with standard silicon-on-insulator (SOI) wafers. Furthermore, note that for a given slot width $w_{\mathrm{S}}$, the width of the arms of the DSW and the PSW has a small influence on the profile of the 
corresponding TE modes, since the field is mainly concentrated on the waveguide slots. Consequently, $w_{\mathrm{s}}$ becomes the key design parameter to be engineered.

(a)
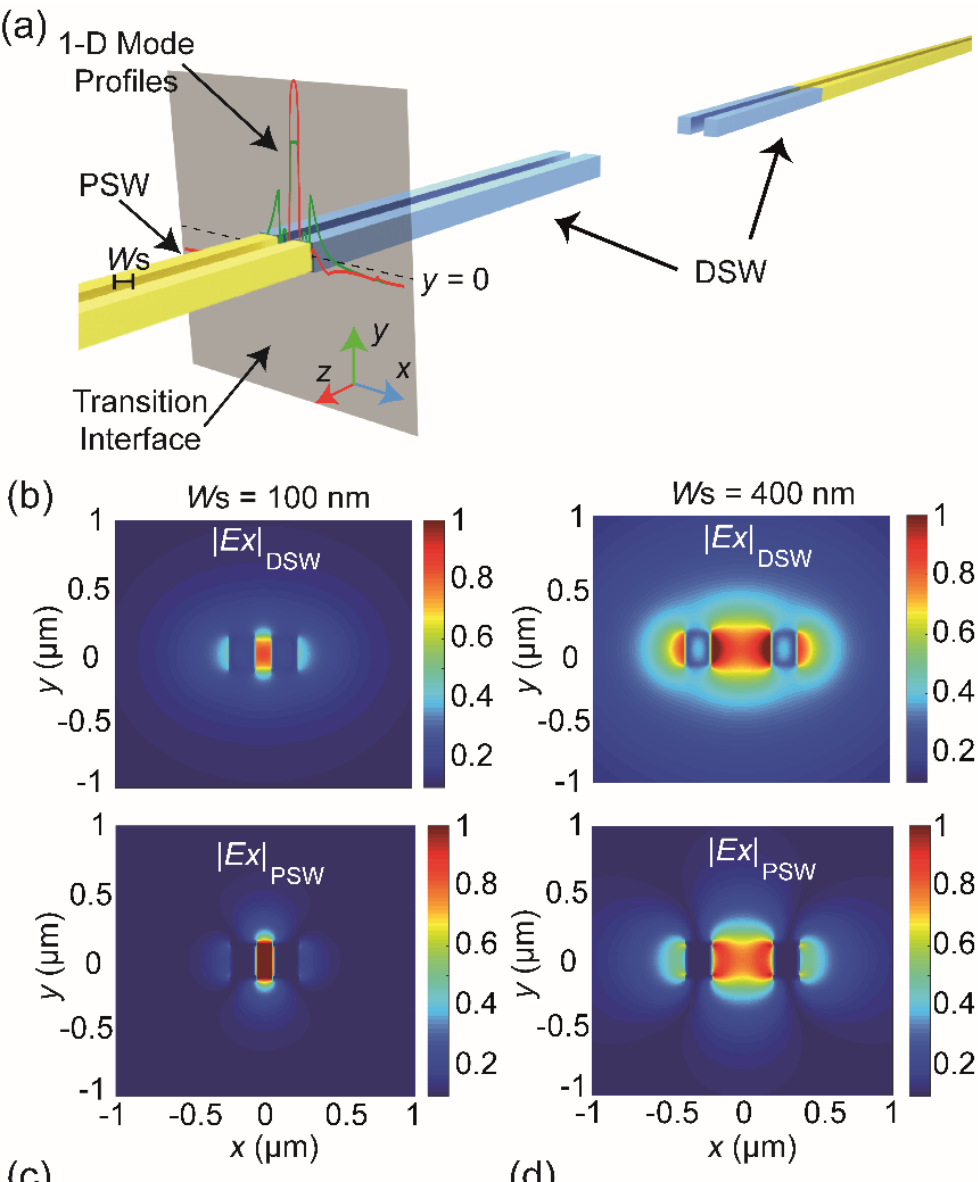

(c)

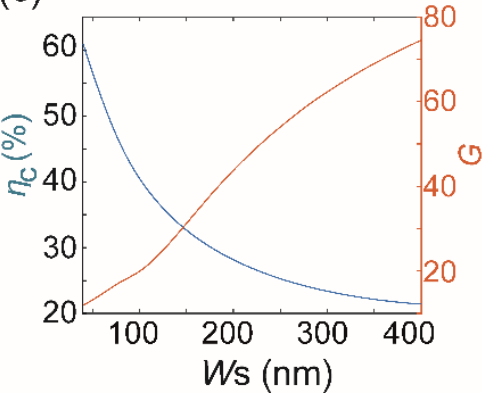

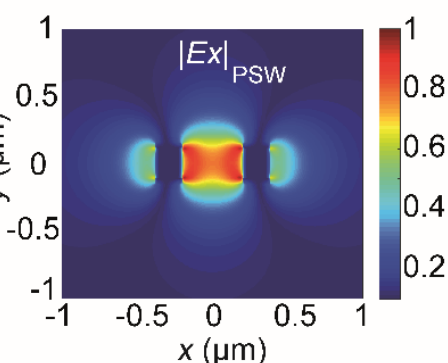

(d)

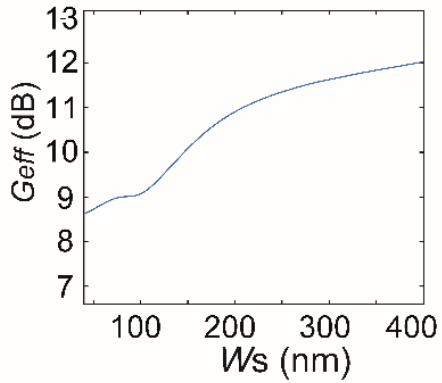

Figure 1. (a) Artwork depicting the coupling between plasmonic and dielectric waveguides. The corresponding mode profiles at $y=$ 0 are displayed on the transition interface (shaded region). (b) E-field intensity distributions of the TE modes excited at the dielectric (top) and plasmonic (bottom) waveguides for $w_{\mathrm{s}}=100 \mathrm{~nm}$ and $w_{\mathrm{s}}=400 \mathrm{~nm}$. Calculations were carried out at the telecom wavelength $\lambda=1550 \mathrm{~nm}$. (c) Coupling efficiency (blue) and DSW-based antenna gain (red) as a function of $w \mathrm{~s}$. The dielectric and metallic waveguides were assumed to be made of $\mathrm{Si}$ and $\mathrm{Au}$, respectively. A Drude model was employed for $\mathrm{Au}$ (21). (d) Effective gain of the DSW-based antenna.

We started by studying the influence of $w_{\mathrm{S}}$ on $\eta_{\mathrm{C}}$, which was numerically calculated using the commercial software CST Microwave Studio. As can be seen in Figure 1d, the fundamental TE modes of both structures are quite similar for low values of $w_{\mathrm{s}}$. However, they become progressively different as $w_{\mathrm{S}}$ increases; the DSW mode becomes rapidly unconfined as $w_{\mathrm{S}}$ increases, whilst the PSW gap plasmon remains concentrated in the slot up to larger values. Consequently, the best figures of $\eta_{\mathrm{C}}$ are retrieved for low values of $w_{\mathrm{S}}$ as shown in Figure $1 \mathrm{~b}$, in consonance with the mode likeness at both structures. 
The next step was to analyze the influence of $w_{\mathrm{S}}$ on $G$. We used Huygens' principle to model this structure as an aperture antenna, following the method typically employed in classic microwave and radiofrequency theory (22). This approach directly links the DSW radiation pattern with the Fourier transform of its mode transverse electric and magnetic fields. A straightforward implication is that less confined modes lead to higher directivities, since the angular extension of the radiation vectors decreases as the spatial extension of the mode increases (6). Figure 1d shows that the DSW fundamental TE mode is less confined as $w_{\mathrm{S}}$ increases, explaining why $G$ grows as a function of this parameter, reaching very high values not achievable with previous plasmonic nanoantennas, see Figure $1 \mathrm{~b}$. It is noteworthy that the directivity of the DSW-based antenna can be easily tuned just by adjusting $w_{\mathrm{S}}$. Moreover, the propagation constant (e.g., $\beta=6.35362 \cdot 10^{6} \mathrm{~m}^{-1}$ for $w_{\mathrm{S}}=180 \mathrm{~nm}$, which provides an effective refractive index $n_{\mathrm{eff}}=1.567$ ) associated with these less confined modes is close to that of the surrounding medium $\left(\mathrm{SiO}_{2}\right.$ with refractive index $\left.n=1.45\right)$, favoring impedance matching and avoiding undesired reflections. Figure $1 \mathrm{c}$ depicts $G_{\text {eff }}$ as a function of $w_{\mathrm{S}}$, showing that there is a large region $\left(w_{\mathrm{S}}>160 \mathrm{~nm}\right)$ for which $G_{\text {eff }}$ is higher than $10 \mathrm{~dB}$, slowly increasing with $w_{\mathrm{S}}$. This feature provides us with an ample design flexibility. That is, $w_{\mathrm{S}}$ can be adjusted to suit applications requiring lower reflections (e.g. to improve source protection) or higher directivities (e.g., to minimize radiation interference with adjacent devices), while keeping a similar performance in terms of overall power efficiency without any coupling structure. Limiting ourselves to typical $w_{\mathrm{S}}$ values $(20-200 \mathrm{~nm}(20),(23))$, we decided to work with $w_{\mathrm{S}}=180 \mathrm{~nm}$, which already shows a gain $G \approx$ 40. Furthermore, we could combine the advantages associated with a small $w_{\mathrm{S}}$ value (enhanced $\eta_{\mathrm{C}}$ ) and a high $w_{\mathrm{S}}$ value (enhanced $G$ ) just by using a simple and compact silicon PSW-DSW transition (see supporting information, section 1), which already yields $\eta_{C}=0.47$ and $G \approx 40$ in a footprint of approximately $1 \mu \mathrm{m}^{2}$.
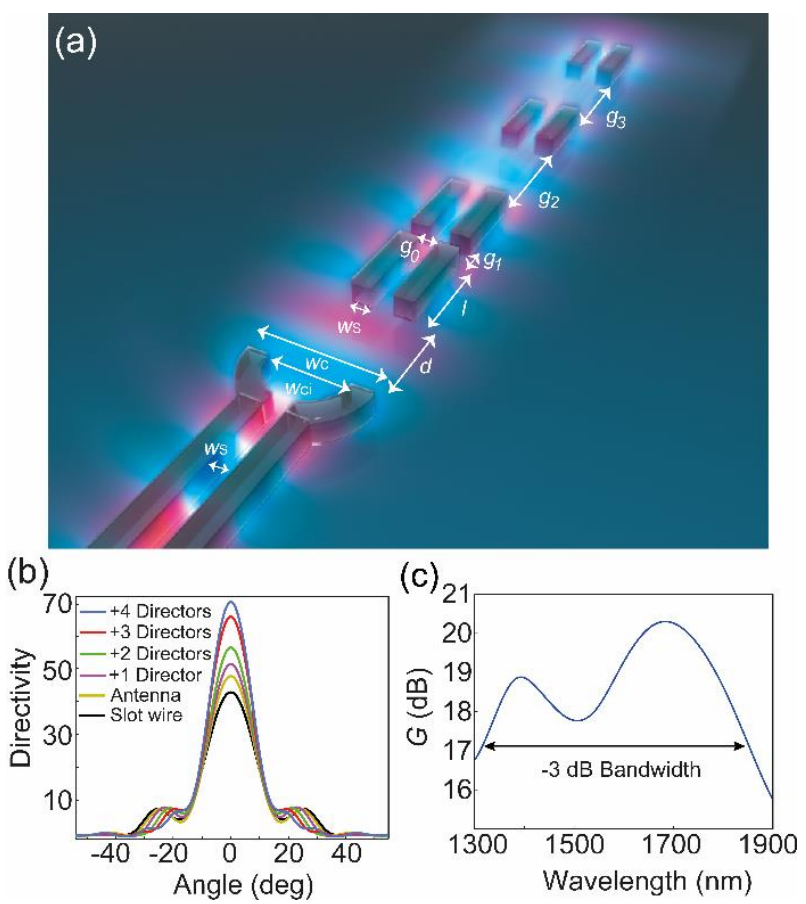

Figure 2. (a) Schematic picture of the nanoantenna and the directors with their corresponding dimensions where $w \mathrm{~s}=180 \mathrm{~nm}, w \mathrm{CI}=$ $640 \mathrm{~nm}, w_{\mathrm{C}}=1000 \mathrm{~nm}, d=500 \mathrm{~nm}, l=800 \mathrm{~nm}, g_{0}=340 \mathrm{~nm}, g_{1}=200 \mathrm{~nm}$, and $g_{2}=g_{3}=1000 \mathrm{~nm}$. CST Microwave Studio was employed to perform the optimization and all the numerical calculations of this work. (b) Simulated directivity of the designed slot waveguide with and without $C$ antenna and directors. (c) Simulated gain of the $C$ antenna. The $-3 \mathrm{~dB}$ Bandwidth is highlighted, demonstrating the broadband behavior of this design.

Note that higher coupling efficiencies could be attained by further optimizing the plasmonic-to-dielectric waveguide transition. For example, a longer taper would approach the $62 \%$ efficiency value for $w_{\mathrm{S}}=40 \mathrm{~nm}$. However, 
although such transitions play an important role in the system, its optimization is out of the scope of this work, which focuses on showing that dielectric antennas can act as high-performance wireless interfaces for plasmonic devices (outperforming previous all-plasmonic approaches), while keeping the design as simple as possible.

Additionally, the directivity can also be further enhanced by modifying the DSW radiating end and by adding some specific extra dielectric elements. In particular, we explored different modifications inspired by V-antenna designs (24), see supporting information, section 2. The best improvement was achieved with a smoothed (C-shaped) version of this kind of structure, see Figure 2a, with a directivity improvement of the order of an $11 \%$, see Figure $2 b$. Moreover, we studied the influence of adding further elements (directors) to the C-antenna design. By analogy with typical metallic Yagi-Uda configurations (25), (26), (27) in which the directors are basically passive antennas of the same type as the principal one (usually dipoles), we considered directors consisting of couples of silicon strips, similar to DSW pieces, see Figure 2a. The number of directors as well as their location and dimensions were optimized through numerical simulations to maximize the directivity in an on-chip footprint as constrained as possible, see supporting information, section 3 . The impact of the $\mathrm{C}$-antenna and a varying number of directors on the directivity is shown in Figure 2b. Our final configuration includes four directors, since additional elements provide small improvements while considerably increasing size, see Figure $2 b$. In this case, a full single antenna (C nanostructure with four directors) achieves a directivity of 71 [previous dielectric nanoparticle antennas reach values up to 12 (16), (17)] and a gain $G=$ 65 at $\lambda=1550 \mathrm{~nm}[G>100$ (linear units) is achieved at longer wavelengths, see Figure 2c]. The corresponding effective gain of this device is $G_{\text {eff }}=0.30 \cdot 65=19.5\left(G_{\text {eff }}=0.47 \cdot 65=30.5\right.$ when using the aforementioned coupler $)$.

Although devices operating at different wavelengths cannot be directly compared (the optical properties of metals are frequency-dependent), we take the work in (11) as a reference (which was designed for a wavelength of $650 \mathrm{~nm}$ ), since, to date (and for the best of our knowledge), it provides the highest efficiency for a plasmonic link in any spectral band, setting the bar for current research in this area. Particularly, the plasmonic nanoantenna designed therein yields a gain of 6.8. Hence, the proposed optical antenna in the present work provides a notable improvement with respect to this previous design. Nonetheless, it is worth mentioning that this improvement comes at the cost of a considerably higher footprint than that of typical plasmonic antennas. Along this line, to compare the overall goodness of different optical antennas, we have defined a figure of merit that takes into account both gain and footprint (see supporting information section 4), according to which, in spite of their larger footprint, the proposed DSW-based antennas clearly outperform previous designs. In addition, we have compared the power efficiency of a plasmonic wired link with that of a wireless one based on the proposed antennas (see supporting information section 5), showing that the wireless scheme exhibits a higher efficiency than the waveguide-based interconnect for distances exceeding $55 \mu \mathrm{m}$ (as an example, the improvements achieved at $100 \mu \mathrm{m}$ and $200 \mu \mathrm{m}$ are higher than 15 and $55 \mathrm{~dB}$, respectively). Moreover, the proposed device possesses a working bandwidth of approximately $600 \mathrm{~nm}$, see Figure 2c, since DSW-based antennas do not rely on resonant phenomena. On the contrary, they display almost perfect impedance matching with the cladding in a wide spectral region (the reflection coefficient of the DSW-based antenna is below 0.03). Thus, unlike in typical plasmonic schemes, auxiliary resonant matching elements (28) are not necessary, avoiding the bandwidth limitation they introduce. 


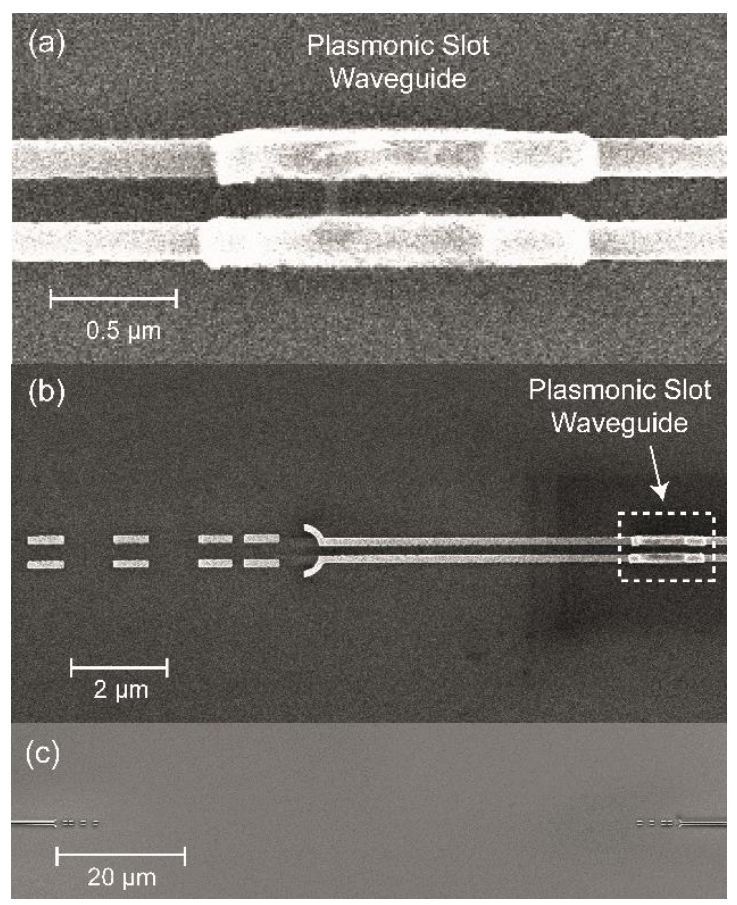

Figure 3. (a) Scanning electron microscope (SEM) image of the fabricated gold slot waveguide section in between the silicon wires. (b) SEM image of the DSW-based antenna connected to a 1- $\mu$ m length plasmonic slot waveguide. (c) SEM image of a 100- $\mu$ m-long link consisting of two antennas as the one shown in (b). All the samples were covered with a 2- $\mu$ m-thick layer of $\mathrm{SiO}_{2}$.

To provide a proof of concept of the proposed approach, we fabricated and characterized different devices using $\mathrm{Au}$ as the constituent material for the plasmonic wires. In the experiments, we inject/collect light to/from the chips via standard silicon grating couplers and waveguides. Therefore, we have a second dielectric-plasmonic transition at each link end (between the exciting/collecting Si waveguide and the corresponding plasmonic waveguide, see Figure 3) as compared to the structures shown in Figure 1, in which a single dielectric-plasmonic transition per link end is assumed (this could actually be the case in a practical application). With this consideration in mind, and following the work in (20), we first fabricated a sample in which a plasmonic slot waveguide section was inserted in between two dielectric slot waveguides (see Figure 3a and Methods), in order to experimentally obtain the value of the PSW-to-DSW coupling efficiency $\eta_{\mathrm{C}}$ (this configuration includes no wireless link). Note that light goes through two PSWto-DSW facets in this structure. Consequently, Fabry-Pérot resonances arising from the presence of the cavity formed by the plasmonic waveguide in between the silicon wires may alter the power transmission efficiency of the system (with respect to a single $\mathrm{Si}-\mathrm{Au}$ transition), depending on $\lambda$ and the cavity length $d_{\mathrm{C}}$. Specifically, with the employed scheme $\left(d_{\mathrm{C}}=1 \mu \mathrm{m}\right)$, the $\mathrm{Si}$-Au-Si transition power efficiency is $\eta_{\mathrm{FP}} \approx$ 0.45 at $\lambda=1550 \mathrm{~nm}$ (or, equivalently, it possesses an insertion loss, IL, of $3.43 \mathrm{~dB}$ ), which is higher than the previously calculated $\eta_{\mathrm{C}}=0.3$ for a single Au-Si transition (as confirmed by the simulations, compare Figures 1c and 4a). The numerically-calculated efficiency $\eta_{\mathrm{FP}}$ of this Si-Au-Si transition (IL $=3.24 \mathrm{~dB}$ ) is in very good agreement with the measurements (see Figure $4 \mathrm{a}$ ). For fixed values of $d_{\mathrm{C}}$ and $\lambda, \eta_{\mathrm{FP}}$ is only a function of $\eta_{\mathrm{C}}$ (and of the theoretically known plasmonic waveguide complex wavenumber). Therefore, the good agreement between the numerically and experimentally obtained IL values for this device at $\lambda=1550$ $\mathrm{nm}$ (Figure $4 \mathrm{a}$ ), implies that $\eta_{\mathrm{C}} \approx 0.3$ is also the coupling efficiency of each single Au-Si transition in the fabricated $\mathrm{Si}-\mathrm{Au}-\mathrm{Si}$ structure (note that we do not directly measure this parameter). The experimental and theoretical values of IL as a function of wavelength for this structure are shown in Figure 4a (the E-field 
distribution of the $\mathrm{Si}-\mathrm{Au}-\mathrm{Si}$ scheme is available in the supporting information, section 6). Notably, the measured insertion loss for a very similar $\mathrm{Si}-\mathrm{Au}-\mathrm{Si}$ scheme based on dielectric strip waveguide to plasmonic slot waveguide transitions (also with a $1-\mu \mathrm{m}$-long plasmonic slot waveguide) was $\mathrm{IL} \approx 14 \mathrm{~dB}$ (20), with a dielectric-to-plasmonic footprint of approximately $8 \mu \mathrm{m}^{2}$ (the coupler footprint is virtually zero in our case).

Next, we fabricated two different samples including full wireless links consisting of two DSW-based antennas, each connected to a 1- $\mu \mathrm{m}$-long plasmonic slot waveguide section, with distances $d$ of 50 and $100 \mu \mathrm{m}$ (see Figures $3 \mathrm{~b}$, $3 \mathrm{c}$ and Methods). For the sake of simplicity, we focused our experimental efforts on the direct butt-coupling between a plasmonic and a dielectric slot wire with $w_{\mathrm{S}}=180 \mathrm{~nm}$, see Figure $3 \mathrm{~b}$ (i.e., we do not include the theoretically-analyzed taper shown in the supporting information, section 1). To test the wireless interconnects, we measured the link efficiency for both distances (50 and $100 \mu \mathrm{m}$ ), attaining a very good agreement with the simulations (see Figure 4b), although small discrepancies arise from waveguide shape imperfections inherent to the fabrication process. A similar behavior to that predicted by the Friis transmission equation (22) can be observed in Figure $4 \mathrm{~b}$ between the 50- and $100-\mu \mathrm{m}$ links, which results in an increase of approximately $6 \mathrm{~dB}$ in the overall link loss as doubling distances between antennas. In addition, we retrieved the antenna gain from the experimental measurements, finding again a good agreement with the numerical results (see Figure 4c). Remarkably, the link is able to radiate with a similar performance within the whole measured optical bandwidth, spanning $50 \mathrm{~nm}$. It must be pointed out that both the experimentallyand numerically-retrieved values of $G_{\text {eff }}$ depicted in Figure 4 for the links including the 1-um-long plasmonic waveguide are slightly higher than the numerically calculated value for a link with single Au-Si transitions, due to the fact that the IL of the Si-Au-Si transition is lower than that of a single Au-Si transition, as mentioned above.
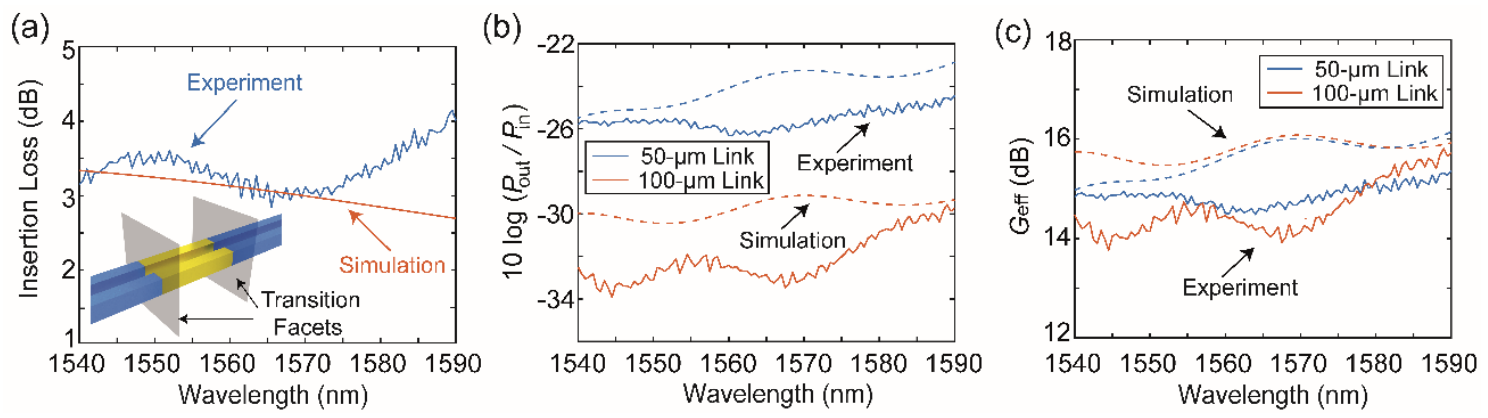

Figure 4. (a) Simulated (red) and experimental (blue) normalized insertion loss of a 1- $\mu$ m length gold slot waveguide. Shaded areas represent the transition facets of the plasmonic wire with the silicon waveguides from which light is injected (collected) via grating couplers, see supporting information, section 7. At a wavelength of $\lambda=1550$, the experimental $\mathrm{IL}=3.43 \mathrm{~dB}$. (b) Comparison of the experimental (solid lines) versus simulated (dashed lines) normalized link power efficiency corresponding to the links with $d=50 \mu \mathrm{m}$ and $d=100 \mu \mathrm{m}$. (c) Comparison of the simulated and measured nanoantenna gain. In the worst case, $G_{\mathrm{eff}}=14.14 \mathrm{~dB}$ at $\lambda=1550 \mathrm{~nm}$. The effective gain was retrieved from the measured power efficiency, which in our experiment is $\eta=G_{\mathrm{eff}^{2}}{ }^{2} \eta_{\mathrm{G}}{ }^{2} \cdot \eta_{\mathrm{PROP}}{ }^{2}$, where $\eta_{\mathrm{G}}$ stands for the experimental grating coupler insertion loss, and $\eta_{\mathrm{PROP}}=\lambda \cdot(4 \pi d)^{-1}$, represents the propagation loss in the link (see supporting information, section 7 for the values of $\eta_{\mathrm{G}}$ ).

Finally, to further test the proposed DSW-based antennas, we carried out an additional experiment in which data streams of $40 \mathrm{Gbit} \cdot \mathrm{s}^{-1}$ were transmitted over $20-\mu \mathrm{m}$-length links (see supporting information, section 8).

Conclusion: We have demonstrated a long-reach wireless plasmonic-dielectric optical interconnect based on silicon nanoantennas able to be directly and efficiently connected to plasmonic systems. Specifically, the numerical study of the modes supported by plasmonic and dielectric slot waveguides with equal slot widths revealed that an efficient direct butt-coupling between these structures is possible. The proper combination of this feature with the development of a new kind of DSW-based nanoantenna proves the utility of these dielectric structures as efficient interfaces for plasmonic devices. In order to test the performance of the proposed nanoantennas, we experimentally demonstrated 
power transmissions at link distances as long as $100 \mu \mathrm{m}$. Despite the fact that the DSW-based antennas here demonstrated exhibit less compact footprints than most of the previous metallic schemes, our hybrid design widely outperforms its plasmonic counterparts in terms of directivity, efficiency and planar reach. Moreover, such a combination merges the ability of silicon nanoantennas to achieve long-reach wireless communications, reconfigurable interconnects, beam-shaping elements, and lab-on-a-chip devices (6), with the advantages of plasmonic structures for ultra-fast data conversion and light concentration and manipulation. Finally, this hybrid configuration might enable the implementation of new sensing applications and microwave plasmonic devices in the THz band (6), (29).

Methods: The fabrication of the dielectric links was based on the use of standard SOI samples from SOITEC wafers with a top silicon layer thickness of $220 \mathrm{~nm}$ (resistivity $\rho \sim 1-10 \Omega \cdot \mathrm{cm}^{-1}$, with a lightly p-type background doping of $\sim 10^{15} \mathrm{~cm}^{-3}$ ). The fabrication is based on an electron beam direct writing process performed on a coated $100 \mathrm{~nm}$ hydrogen silsesquioxane (HSQ) resist film. The mentioned electron beam exposure, performed with a Raith150 tool, was optimized in order to reach the required dimensions employing an acceleration voltage of $30 \mathrm{KeV}$ and an aperture size of $30 \mu \mathrm{m}$. After developing the HSQ resist using TetraMethylAmmoniumHyrdoxide (TMAH) as developer, the resist patterns were transferred into the SOI samples employing an optimized Inductively Coupled Plasma- Reactive Ion Etching (ICP-RIE) process with fluoride gases $\left(\mathrm{SF}_{6}\right.$ and $\left.\mathrm{CF}_{4}\right)$. Once the dielectric waveguides and antennas were fabricated, the plasmonic structures were placed inside the silicon waveguides gaps by means of a second e-beam lithography process. This new lithography was performed again with the Raith150 tool ( $10 \mathrm{KeV}$, aperture $30 \mu \mathrm{m})$ prior to a new metal evaporation ( $220 \mathrm{~nm}$ of gold $+5 \mathrm{~nm}$ of titanium for adhesion enhancement) performed with a Pfeiffer Classic 500 tool. Finally, a soft lift-off process (leaving the sample in acetone for 8 hours) was used to define the plasmonic waveguides. To ensure the right positioning of the plasmonic structures, different alignment marks were created in the previous silicon etching level, guiding us in an iterative exposure process to achieve the best alignment. The plasmonic structures placement during final exposure was adjusted according to this iterative process in order to compensate e-beam drifts. Finally, a $\mathrm{SiO}_{2}$ upper cladding (2- $\mu \mathrm{m}$ thickness) was deposited atop the SOI sample by Plasma Enhanced Chemical Vapor Deposition (PECVD) performed with a Centura 5200 tool from Applied Materials.

\section{Associated Content:}

Supporting Information

Section 1. Silicon coupler for enhanced coupling efficiency. Section 2. Modelling of the C-Shaped antenna. Section 3. Design of antenna directors. Section 4. Power efficiency of wireless versus plasmonic links. Section 5. Figure of merit of the wireless interconnect. Section 6. E-field distribution in the Si-Au-Si transition. Section 7. Experimental grating coupling efficiency. Section 8. Data transmission experimental test.

Acknowledgement: This work was supported by project TEC2015-73581-JIN PHUTURE (AEI/FEDER, UE) and Generalitat Valenciana's PROMETEO grant NANOMET PLUS (PROMETEO II/2014/34).

\section{Conflict of Interest:}

The authors declare no competing financial interest. 


\section{References}

(1) Brongersma, M. L.; Shalaev, V. M. The Case for Plasmonics. Science 2010, 328 (5977), 440-441.

(2) Haffner, C.; Heni, W.; Fedoryshyn, Y.; Niegemann, J.; Melikyan, A.; Elder, D. L.; Baeuerle, B.; Salamin, Y.; Josten, A.; Koch, U.; Hoessbacher, C.; Ducry, F.; Juchli, L.; Emboras, A.; Hillerkuss, D.; Kohl, M.; Dalton, L. R.; Hafner, C.; Leuthold, J. All-plasmonic Mach-Zehnder modulator enabling optical high-speed communication at the microscale. Nat. Photonics 2015, 9 (7), 525-528 .

(3) Alù, A.; Engheta, N. Wireless at the Nanoscale: Optical Interconnects using Matched Nanoantennas. Phys. Rev. Lett. 2010, 104 (21), 213902.

(4) Khurgin, J. B. How to deal with the loss in plasmonics and metamaterials. Nat. Nanotech. 2015, 10, $2-6$.

(5) Briggs, R. M.; Grandidier, J.; Burgos, S. P.; Feigenbaum, E.; Atwater, H. A. Efficient Coupling between Dielectric-Loaded Plasmonic and Silicon Photonic Waveguides. Nano Lett. 2010, 10 (12), 4851-4857.

(6) García-Meca, C.; Lechago, S.; Brimont, A.; Griol, A.; Mas, S.; Sánchez, L.; Bellieres, L.; Losilla, N. S.; Martí, J. On-chip wireless silicon photonics: from reconfigurable interconnects to lab-on-chip devices. Light Sci. Appl. 2017, 6, e17053.

(7) Novotny, L.; van Hulst, N. Antennas for light. Nat. Photonics 2011, 5 (2), 83-90.

(8) Bharadwaj, P.; Deutsch, B.; Novotny, L. Optical Antennas. Adv. Opt. Photon. 2009, 1 (3), $438-483$.

(9) Ni, X.; Emani, N. K.; Kildishev, A. V.; Boltasseva, A.; Shalaev, V. M. Broadband Light Bending with Plasmonic Nanoantennas. Science 2012, 335 (6067), 427.

(10) Dregely, D.; Lindfors, K.; Lippitz, M.; Engheta, N.; Totzeck, M.; Giessen, H. Imaging and steering an optical wireless nanoantenna link. Nat. Commun. 2014, 5, 4354.

(11) Yang, Y.; Li Q.; Qiu, M. Broadband nanophotonic wireless links and networks using on-chip integrated plasmonic antennas. Sci. Rep. 2016, 6, 19490.

(12) Zhang, Y. S.; Watts, B. R.; Guo, T. Y.; Zhang, Z. Y.; Xu C. Q.; Fang, Q. Optofluidic Device Based Microflow Cytometers for Particle/Cell Detection: A Review. Micromachines 2016, 7 (4), 70.

(13) Lechago, S.; García-Meca, C.; Sánchez-Losilla, N.; Griol, A.; Martí, J. High signal-to-noise ratio ultracompact lab-on-a-chip microflow cytometer enabled by silicon optical antennas. Opt. Express 2018, 26 (20), 25645-25656.

(14) Solís, D. M.; Taboada, J. M.; Obelleiro, F.; Landesa, L. Optimization of an optical wireless nanolink using directive nanoantennas. Opt. Express 2013, 21 (2), 2369-2377.

(15) Zhao, Y.; Engheta, N.; Alù, A. Effects of shape and loading of optical nanoantennas on their sensitivity and radiation properties. J. Opt. Soc. Am. B 2011, 28 (5), 1266.

(16) Filonov, D. S.; Krasnok, A. E.; Slobozhanyuk, A. P.; Kapitanova, P. V.; Nenasheva, E. A.; Kivshar, Y. S.; Belov, P. A. Experimental verification of the concept of all-dielectric nanoantennas. Appl. Phys. Lett. 2012, 100 (20), 201113.

(17) Krasnok, A. E.; Miroshnichenko, A. E.; Belov, P. A.; Kivshar, Y. S. All-dielectric optical nanoantennas. Opt. Express 2012, 20 (18), 20599-20604.

(18) Van Acoleyen, K.; Rogier, H.; Baets, R. Two-dimensional optical phased array antenna on silicon-oninsulator. Opt. Express 2010, 18 (13), 13655-13660.

(19) Sun, J.; Timurdogan, E.; Yaacobi, A.; Hosseini, E. S.; Watts, M. R. Large-scale nanophotonic phased array. Nature 2013, 493 (1), 195-199.

(20) Tian, J.; Yu, S.; Yan, W.; Qiu, M. Broadband high-efficiency surface-plasmon-polariton coupler with siliconmetal interface. Appl. Phys. Lett. 2009, 95 (1), 013504.

(21) Johnson P. B.; Christy, R. W. Optical Constants of the Noble Metals. Phys. Rev. B 1972, 6 (12), 4370.

(22) Balanis, C. Antenna Theory: Analysis and Design; Eds.; John Wiley \& Sons, Inc.: New Jersey, 2005; pp 94 96.

(23) Veronis, G.; Fan, S. Modes of Subwavelength Plasmonic Slot Waveguides. J. Light. Technol. 2007, 25 (9), 2511-2521.

(24) Klemm. M. Novel Directional Nanoantennas for Single-Emitter Sources and Wireless Nano-Links. Int. J. Opt. 2012, 348306.

(25) Curto, A. G.; Volpe, G.; Taminiau, T. H.; Kreuzer, M. P.; Quidant, R.; van Hulst, N. F. Unidirectional Emission of a Quantum Dot Coupled to a Nanoantenna. Science 2010, 329 (5994), 930-933. 
(26) Dregely, D.; Taubert, R.; Dorfmüller, J.; Vogelgesang, R.; Kern, K.; Giessen, H. 3D optical Yagi-Uda nanoantenna array. Nat. Commun. 2011, 2, 267.

(27) Coenen, T.; Vesseur, E. J. R.; Polman, A.; Koenderink, A. F. Directional Emission from Plasmonic YagiUda Antennas Probed by Angle-Resolved Cathodoluminescence Spectroscopy. Nano Lett. 2011, 11 (9), 3779-3784.

(28) Alù, A.; Engheta, N. Input Impedance, Nanocircuit Loading, and Radiation Tuning of Optical Nanoantennas. Phys. Rev. Lett. 2008, 101, 043901.

(29) Burla, M., Bonjour, R.; Salamin, Y.; Haffner, C.; Heni, W.; Hosessbacher, C.; Fedoryshyn, Y.; Abrecht, F. C.; Johnston, P. V.; Elder, D. L.; Dalton, L. R.; Leuthold, J.. 2016 IEEE International Topical Meeting on Microwave Photonics (MWP), 2016, 259. 


\title{
For Table of Contents Use Only
}

\section{All-Silicon On-chip Optical Nanoantennas \\ As Efficient Interfaces for Plasmonic Devices}

\author{
Sergio Lechago *, Carlos García-Meca, Amadeu Griol, Miroslavna \\ Kovylina, Laurent Bellieres and Javier Martí
}

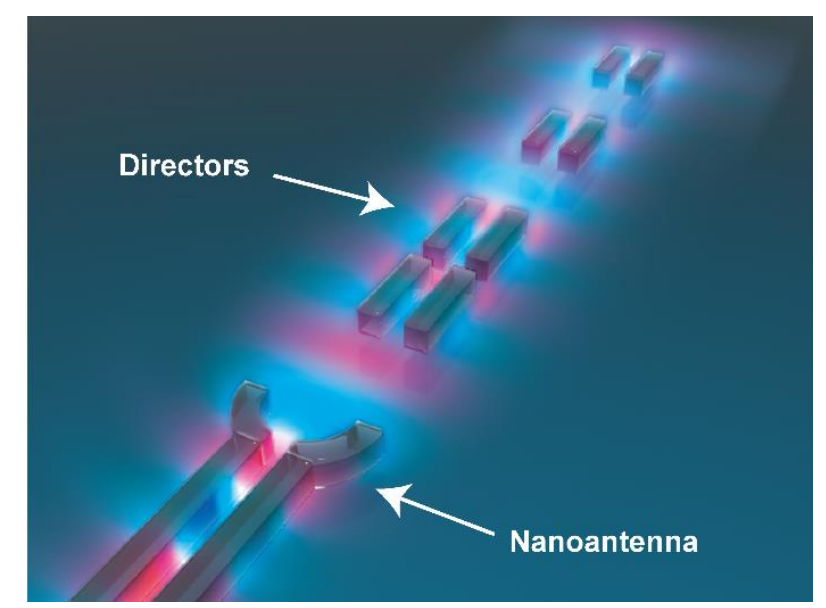

The picture represents an on-chip dielectric C-shaped antenna, which is the key building block to achieve high-performance plasmonic-dielectric wireless interconnects. The electromagnetic field of the aforementioned antenna is also depicted in the artwork. 